\title{
Preliminary study on exploration of seaweed for an alternative to antibiotic growth promoter in poultry feed additives
}

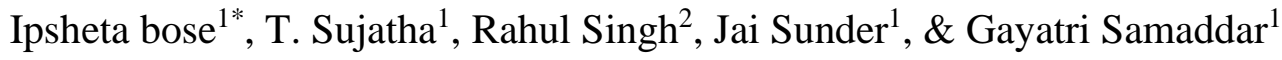 \\ ${ }^{1}$ ICAR-CIARI, Port Blair, India-744101 \\ ${ }^{2}$ Integral University, Lucknow, India-2260241 \\ *ipsheta18@gmail.com
}

\begin{abstract}
Seaweed (known as the marine algae) has a tradition of being part of the animal feed in the coastal areas, from ancient times. Seaweed, are mixed with animal feed, because when consumed alone can have some negative effects on the animal. Seaweed are very rich in useful metabolite (pigments, carotenoids, phlorotannin, polyunsaturated fatty acids, agar, alginate and carrageenan) and minerals (iodine, zinc sodium calcium, magnesium, iron, selenium), being considered as a natural source of additives that can substitute the antibiotic usage in various animals. Inclusion of naturally available material in poultry feed additive can have a positive influence on the poultry performance and enrich the quality of their by product. As an easily available and renewable biomass rich in biologically active compounds, seaweeds can meet this demand. Different forms of seaweed powder from naturally occurring biomass, cultivated or waste biomass, extracted compounds, post extraction residues or liquid extracts may be used in poultry feeding. This study describes the nutritional values of seaweeds and their effects in poultry feed additive, and to what extent their unique properties can serve as an alternative to antibody growth promoter
\end{abstract}

\section{INTRODUCTION}

India has vast resource of livestock and poultry, which play a vital role in improving the socioeconomic condition and National economy. Andaman and Nicobar Island is rich in its diversity and natural resources. Island's agriculture and animal husbandry activities are barely 150 years old. Till late 18 th century islands were inhabited by only native tribal and the major food animals was pigs and birds. With the settlement of penal colony by Britishers, livestock farming came in existence to meet out the demand of meat and milk. The island is endowed with rich faunal diversity and among them the farm animal genetic resources comprises of cattle, goat, buffalo, pig and poultry.

Poultry production is an important area of the agricultural economy. Nowadays, there is an interest in novel sources of feed additives that will improve production performance and poultry health. Rural poultry are of prime important and of great concern for the poor rural farmers and tribal farming community of these islands as it is the only source of egg for them. And if we look at the worldwide growing demand of poultry production has resulted in pressure on breeder, growers and also nutritionists to increase the growth rate of birds and the feed 
efficiency. Moreover, the shift toward further processed products has emphasized the necessity for higher standards in poultry meat to improve sensory characteristics and functional property.

If we talk about the situation of the present global poultry sector, there are numerous problems faced by the sector and there will be number of challenges in the future trends as well, which will emphasis mainly on four areas such as food security, social challenges (poverty alleviation and equity), health (animal and human) and environment (natural resources and climate change)(Anne Mottet and G. Tempio, 2017) ${ }^{1}$. Poultry is the fastest growing agricultural subsector, especially in developing countries. It makes a substantial contribution to food security and nutrition, providing energy, protein, and essential micro-nutrients to humans, with short production cycles and the ability to convert a wide range of agri-food by-products and wastes into meat and eggs edible by humans.

Managing production cost while maintaining an efficient animal production can be challenging. Better nutrients digestion and absorption improves animal growth and contributes in this way to a more profitable production of animal protein products. In addition, increasing global pressure on animal protein standards emphasis importance of quality and composition of meat and egg entering the market. However, poultry represent a threat to human health, especially as a vector of infectious diseases and because of its role in antimicrobial resistance. On the other hand, in animal agriculture antibiotics are often used to treat various pathogenic diseases in poultry livestock. It is used to increase the production level both quality wise and quantity wise. One such synthetic hormone used for poultry production is melatonin. Melatonin is one of the important hormones that prevent metabolic and physiological disorders in poultry. Melatonin helps regulate the biological process of the system such as excretion, respiration, reproduction, circulation and with the immune system (Suleyman CALISLAR, et al., 2018) ${ }^{2}$. In poultry it helps with their pattern of feed consumption, body heat and energy metabolism. Melatonin stimulates the secretion of growth hormone and thus positively effects the growth performance of the poultry (Yaxiong Jia et al., 2016) ${ }^{3}$. However excessive use of antibiotics results in development of drug-resistant bacterial pathogen. This shows the urgency to find out some essential alternatives to treat these diseases so that the heath of poultry is improved which will ultimately enhance the safety of poultry product. As an easily available and renewable biomass rich in biologically active compounds, seaweeds can meet these emerging demands to improve the poultry farming and production system of this island. Seaweed or macro algae generally reside in littoral zone and are found in different shapes, size, color and species. Seaweed has a long history of use as livestock feed. Seaweed has plenty of essential nutrients, especially trace elements and several other bioactive substances. Seaweed is highly rich in antioxidants, which protects the body from damages caused by free radicals and protects the cell from their impacts (Times of India, June 22, 2020) ${ }^{4}$, free radical contributes to many health related issue, and seaweed is rich in carotenoids and flavonoids which helps eliminating the free radicals. Seaweed is also rich in iodine and is good source of meeting daily iodine requirements (Leslie Mendoza Temple et al., hypothyroidism, Integrative Medicine (Fourth Edition), 2018) ${ }^{5}$. Seaweed are rich source of pigments namely chlorophyll, carotenoids, and phycobiliproteins, carotenoids are mainly found in chloroplast and chromoplast of seaweed (Hemaiswarya Shanmugam et al., 2018) ${ }^{6}$. 
It is very rich in useful metabolites (pigments, carotenoids, phlorotannin, polyunsaturated fatty acids, agar, alginate and carrageenan) and minerals (iodine, zinc, sodium, calcium, manganese, iron, selenium) (Tiago Morais et al., 2020) ${ }^{7}$. Seaweed is also known for its antimicrobial property. In a study conducted on antibacterial effect of seaweed it was concluded that seaweed as a feed could reduce the effective required doses of antibiotics (Garima Kulshreshtha et al.,2020) ${ }^{8}$, hence the consumption of antibiotics in commercial poultry farms can be reduced, thus further eliminating or reducing the drug resistant bacteria in poultry livestock.

\section{MATERIAL AND METHODS}

This experimental study entitled Preliminary study on exploration of seaweed for an alternative to antibiotic growth promoter in poultry feed additive was conducted in the Division of Animal Science, ICAR- Central Island Agriculture Research Institute, Port Blair, Andaman And Nicobar Island.

Sample Preparation

The Brown seaweed samples (Sargassum spp.) were freshly collected from the sea of South Andaman. Seaweed were washed and cleaned with running tap water to remove all the sand and extraneous substances. After cleaning the seaweed were dried in two different groups i.e. shadow-dried and autoclaved dried followed by drying in room temperature, the sample took 4-5 days to completely get dried. The dried samples from both the group were cut into small pieces. $100 \mathrm{~g}$ of dried sample from each group were extracted with $800 \mathrm{ml}$ of distilled water using a dilution ratio of 1:8. The prepared dilution was overnight stored in shaker incubator. The samples were then ground in a grinder to obtain a mash form. Finally, the mash was filtered to remove the insoluble material.

Experimental design and feeding trail

A total of 28 Vanaraja Chicks with 24 laying and 4 male chicks were used in a 15 days trial. All the birds were randomly assigned for four different treatment group, 7 birds per group (6 female and 1 male chicken). The chickens were provided the seaweed extract as water feed along with basil diet. The treatment comprises of;

T1: water supplementation of $5 \mathrm{ml}$ sundried aqueous extract per chicken.

T2: water supplementation of $5 \mathrm{ml}$ autoclave aqueous extract per chicken.

T3: $0.1 \mathrm{ml}$ melatonin was injected to each bird.

T4: control without any supplementation. 


\section{SAMPLING AND MEASUREMENT}

\section{Hematological Parameter}

After feeding for around 1 week, $5 \mathrm{ml}$ blood samples were collected from branchial vein of 3 birds/treatment using a sterilized syringe and stored in vacutainer containing EDTA. The collected blood samples were taken to the laboratory and stored at $4^{\circ} \mathrm{C}$. The hemoglobin determination was made with a Sahli's Hemoglobinometer, and the reading was estimated using comparator block.

\section{Oxidative Stress Parameter}

Blood sample were collected from all 28 birds after one week of feeding in a vacutainer containing heparin, and then were immediately centrifuged at $2000 \mathrm{rpm}$ for $15 \mathrm{~min}$. Each serum after centrifugation was stored at $-20^{\circ} \mathrm{C}$ until use, these sera was then used for quantifying free radical test with TBARS and NBT assay. For the determination of TBARS, a slightly modified assay of (Papadopoulou, A., et al., 2017) ${ }^{9}$ was used. According to this method 50 $\mu$ l sample was made-up to $1 \mathrm{ml}$ with distilled $\mathrm{H}_{2} \mathrm{O}$, along with that $50 \%$ TCA, $0.375 \%$ TBA and $11.5 \mathrm{~N} \mathrm{HCL}$ was placed in water bath for 1 hour at $99^{\circ} \mathrm{C}$. Then the sample was kept for cooling in room temperature for $10 \mathrm{~min}$. The samples were centrifuged at $2000 \mathrm{rpm}$ for $15 \mathrm{~min}$ and the absorbance of the supernatant was read at $535 \mathrm{~nm}$ using spectrophotometer.

The determination of free radical activity was based on the method of Nitroblue Tetrazolium assay (NBT) according to (Stefaniak, T., et al., 2019) ${ }^{10}$. A total of $50 \mu \mathrm{l}$ sample was taken and mixed with $50 \mu \mathrm{l}$ of $0.1 \%$ NBT. The prepared sample were incubated for $40 \mathrm{~min}$ at $37^{\circ} \mathrm{C}$ followed by washing it with $1 \mathrm{X}$ PBS. After discarding the supernatant, $50 \mu \mathrm{l}$ of pellet from each sample content was taken in the test tubes containing $2 \mathrm{KOH}$ and DMSO, $120 \mu \mathrm{l}$ and $140 \mu \mathrm{l}$ respectively. Finally, readings were observed using the ELISA at $630 \mathrm{~nm}$.

Humoral Immune Response

Humoral immune response was assessed for screening the immunity status of the chicken against goat RBC. The suspension of goat RBC was used as an antigen, and was prepared (2\% $\mathrm{v} / \mathrm{v})$ in PBS and was injected into the wings vein of the bird $(0.2 \mathrm{ml}$ per bird). For making the RBC suspension blood was collected from healthy goat. About $5 \mathrm{ml}$ blood was collected in Anticoagulant Alsever's solution (1:1 ratio). The prepared samples were Centrifuged at 2000 rpm for $10 \mathrm{~min}$ Then the red blood cells were Washed three times in 50ml PBS 50 $\mu 1$ of phosphate buffer saline (PBS). After final wash the pellet was stored at $4^{\circ} \mathrm{C}$. Then each chick was injected intravenously with $0.2 \mathrm{ml}$ of GRBC. The blood samples were taken after 1 week of feeding from the wing vein of the chick and the sera was separated out using cooling centrifuged at 4000 r.p.m. for 10 mins. The harvested serum was later stored at $4^{\circ} \mathrm{C}$. Total antibody titre of antibodies against GRBC was determined as per described by (Martin 1989) ${ }^{11}$ using Hemagglutination test. 
Sensory parameter of Egg

After 15 days of feeding, eggs were collected from the different feed groups and evaluation of different feed source and their influence on egg yolk color was determined. In this the simple descriptive visual observation was used on the basis of light yellow, bright yellow, highly pigmented for categorizing the scale of color or pigment in the yolk.

\section{RESULT AND DISCUSSION}

\section{Blood Profile (Hemoglobin Content)}

Effect of crude seaweed extract supplementation on hemoglobin content is given in the table below. The blood was collected after 15 days of feeding, and from each group 3 samples were collected. As tabulated in Table 1, all the groups have considerably similar statistical values, but if we look at the cumulative result melatonin (synthetic drug) had the maximum hemoglobin content, followed by aqueous autoclave had the second highest average content of hemoglobin. Likewise, it was reported, that hot water extraction of sargassum spp. fed to rainbow trout (Oncorhynchus mykiss) resulted in affecting both RBCs and WBCs counts, hematocrit, and hemoglobin concentration compared with the control, resulting rise in hemoglobin level (Zeraatpisheh et al.,2018) ${ }^{12}$.

\begin{tabular}{|c|c|c|c|}
\hline \multirow{2}{*}{ GROUPS } & \multicolumn{3}{|c|}{ HEMOGLOBIN CONTENT } \\
& 1 & 2 & 3 \\
\hline SHADOW-DRIED & $12 \pm 0.12$ & $10 \pm 0.23$ & $10 \pm 0.10$ \\
\hline AUTOCLAVE-DRIED & $11.4 \pm 0.26$ & $10 \pm 0.10$ & $11.3 \pm 0.25$ \\
\hline MELATONIN & $11.4 \pm 0.26$ & $14.4 \pm 0.28$ & $12 \pm 0.12$ \\
\hline CONTROL & $10 \pm 0.10$ & $9 \pm 0.09$ & $11 \pm 0.11$ \\
\hline
\end{tabular}

Table 1: Different feeding group and hemoglobin level in chicken blood

Free Radical Stress

Lipid peroxidation was determined based on malondialdehyde (MDA) level, measured as an amount of thiobarbituric acid reactive substance (TBARS) followed by spectrophotometer reading at 535n. The graph from figure 1 . depicts that TBARS values of autoclaved aqueous extract supplemented group substantially decreased, whereas the values for shadow-dried and melatonin groups were significantly higher than that of the autoclave-dried group. This result indicates that the autoclaved-dried aqueous extract can be considered an effective feed additive solution to prolong oxidative stability with antioxidant enrichment. 


\begin{tabular}{|c|c|c|c|}
\hline $\begin{array}{c}\text { shadow- } \\
\text { dried }\end{array}$ & autoclave & melatonin & control \\
\hline $1.73 \pm 0.17$ & $1.42 \pm 0.11$ & $1.09 \pm 0.01$ & $1.82 \pm 0.11$ \\
\hline $2.03 \pm 0.18$ & $1.32 \pm 0.23$ & $1.11 \pm 0.11$ & $1.4 \pm 0.16$ \\
\hline $1.84 \pm 0.17$ & $1.05 \pm 015$ & $1.51 \pm 0.19$ & $1.44 \pm 0.07$ \\
\hline $2.07 \pm 0.19$ & $1.21 \pm 0.67$ & $1.53 \pm 0.21$ & $0.88 \pm 0.19$ \\
\hline $1.73 \pm 0.04$ & $0.94 \pm 0.01$ & $1.78 \pm 0.20$ & $2.42 \pm 0.20$ \\
\hline $1.67 \pm 0.1$ & $1.4 \pm 0.28$ & $1.07 \pm 0.17$ & $1.53 \pm 0.10$ \\
\hline $1.65 \pm 0.03$ & $1.42 \pm 0.14$ & $1.8 \pm 0.15$ & $1.03 \pm 0.17$ \\
\hline
\end{tabular}

Table 2: TBARS count of different feeding groups

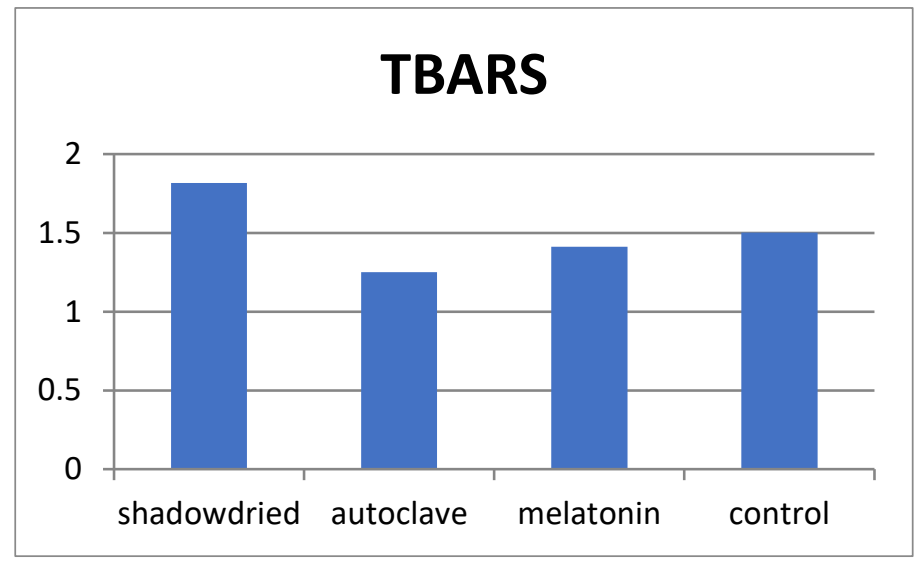

Figure 1: Graph showing free-radical count using TBARS assay

The NBT result is tabulated in table 3 and the graph obtain from these values shows a remarkable declining status in melatonin group. The autoclaved-dried aqueous extract had lower NBT value compared to shadow-dried and the control group. In a study it is proved that the seaweed is rich in antioxidant properties, thus it creates a protective barrier against the oxidative stress (Begum, R., et al., 2021) ${ }^{13}$. Therefore, in this research it can be concluded that seaweed contain some antioxidant properties due to which free-radicals were less in the treatment group. Hence the autoclaved-dried aqueous extract of Sargassum species of seaweed showed positive effect against the oxidative stress but proved less effective than melatonin group. No accurate prior report of the effect of brown sea weed (Sargassum spp.) extract on free radical count could be traced, but the current study revealed, a notable reduction on the free radical count on feeding autoclaved dried aqueous extract of the Sargassum spp. of seaweed at the dilution ration of 1:8. 


\begin{tabular}{|c|c|c|c|}
\hline $\begin{array}{c}\text { shadow- } \\
\text { dried }\end{array}$ & autoclave & melatonin & control \\
\hline $0.697 \pm 0.05$ & $0.605 \pm 0.039$ & $0.644 \pm 0.069$ & $0.708 \pm 0.144$ \\
\hline $0.685 \pm 0.056$ & $0.641 \pm 0.036$ & $0.723 \pm 0.075$ & $0.676 \pm 0.158$ \\
\hline $0.667 \pm 0.063$ & $0.699 \pm 0.039$ & $0.693 \pm 0.062$ & $1.007 \pm 0.176$ \\
\hline $0.765 \pm 0.072$ & $0.66 \pm 0.039$ & $0.594 \pm 0.039$ & $0.676 \pm 0.081$ \\
\hline $0.629 \pm 0.056$ & $0.628 \pm 0.047$ & $0.616 \pm 0.046$ & $0.575 \pm 0.096$ \\
\hline $0.612 \pm 0.074$ & $0.71 \pm 0.059$ & $0.571 \pm 0.033$ & $0.747 \pm 0.114$ \\
\hline $0.717 \pm 0.080$ & $0.626 \pm 0.0048$ & $0.523 \pm 0.015$ & $0.585 \pm 0.088$ \\
\hline
\end{tabular}

Table 3: NBT values of chicken blood serum from different feeding groups

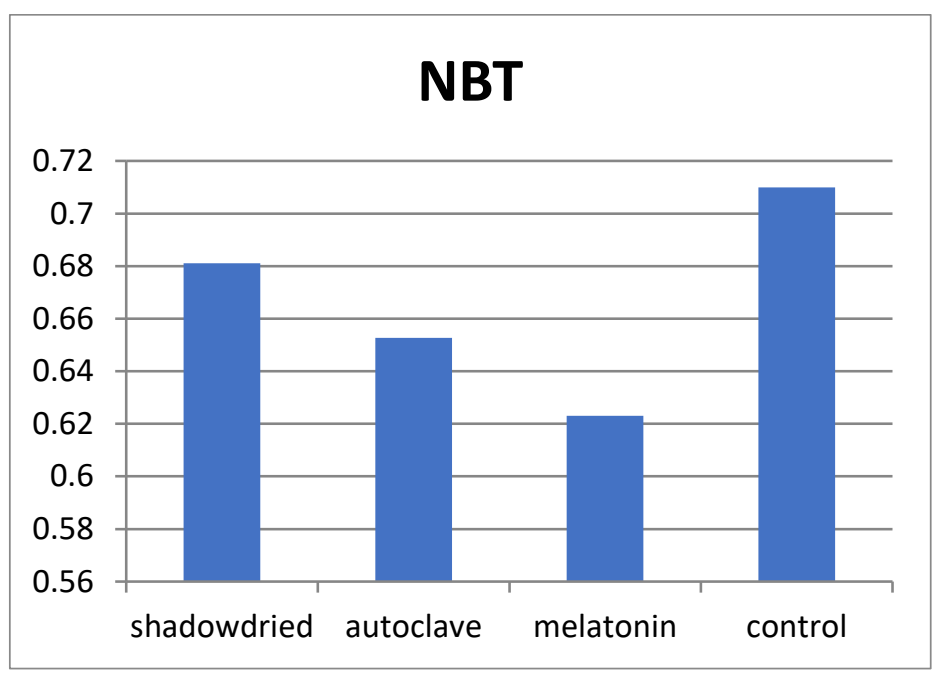

Figure 2: Graph showing free-radical count using NBT assay

Humoral Immunity

GRBC are T-cell dependent antigen and were used to measure the humoral immune response. The antibody response to GRBC indicates the ability of the bird to produce antibody (Arun Kumar De at al., 03 April 2012) ${ }^{14}$. The antibody titer in the serum of individual chickens was determined by hemagglutination (HA) test. The HA titer was expressed as the $\log _{2}$ of the reciprocal of the highest dilution which showed complete hemagglutination. The difference between the treatment were analyzed using one-way analysis of variance (ANOVA). The result of immune response (humoral) assessed through HA method are presented in figure 3 and the graphs illustrates a slight variation among autoclave and melatonin group, both the group had 
no major difference but autoclave group was slightly superior, and among all the other group control group i.e. the non-treatment group had the least antibody level.

(Quingchao Shi et al., 2019) ${ }^{15}$ studied about supplementing black sargassum sp. Diet, and discovered a significant enhancement of immune response in species with optimum supplementation level. (Qadri et al., 2019) ${ }^{16}$ reported that seaweed extract when given to a set of poultry chicken, their immune response was consistently improved, that shows that seaweed contain essential bioactive compounds which is beneficial for the immune system. In accord with their results, the autoclaved dried sargassum spp. of seaweed was found to be much more effective in increasing the immunity of the birds in the current study.

\begin{tabular}{|c|c|c|c|}
\hline shadow dried & autoclave & melatonin & control \\
\hline $0.60206 \pm 0.23$ & $0.60206 \pm 0.45$ & $1.20412 \pm 0.23$ & $1.20412 \pm 0.45$ \\
\hline $1.20412 \pm 0.35$ & $1.20412 \pm 0.45$ & $1.20412 \pm 0.36$ & $1.20412 \pm 0.21$ \\
\hline $1.20412 \pm 0.40$ & $0.60206 \pm 0.39$ & $1.20412 \pm 0.38$ & $1.20412 \pm 0.53$ \\
\hline $1.20412 \pm 0.56$ & 0 & $1.20412 \pm 0.35$ & $0.30103 \pm 0.53$ \\
\hline $1.20412 \pm 0.27$ & $0.90309 \pm 0.33$ & $0.90309 \pm 0.27$ & $1.80618 \pm 0.50$ \\
\hline $0.90309 \pm 0.22$ & $1.20412 \pm 0.31$ & $0.90309 \pm 0.23$ & $0.90309 \pm 0.28$ \\
\hline $0.90309 \pm 0.22$ & $0.30103 \pm 0.06$ & $0.60206 \pm 0.12$ & $1.20412 \pm 0.29$ \\
\hline
\end{tabular}

Table 4: Anti-GRBC HA titres

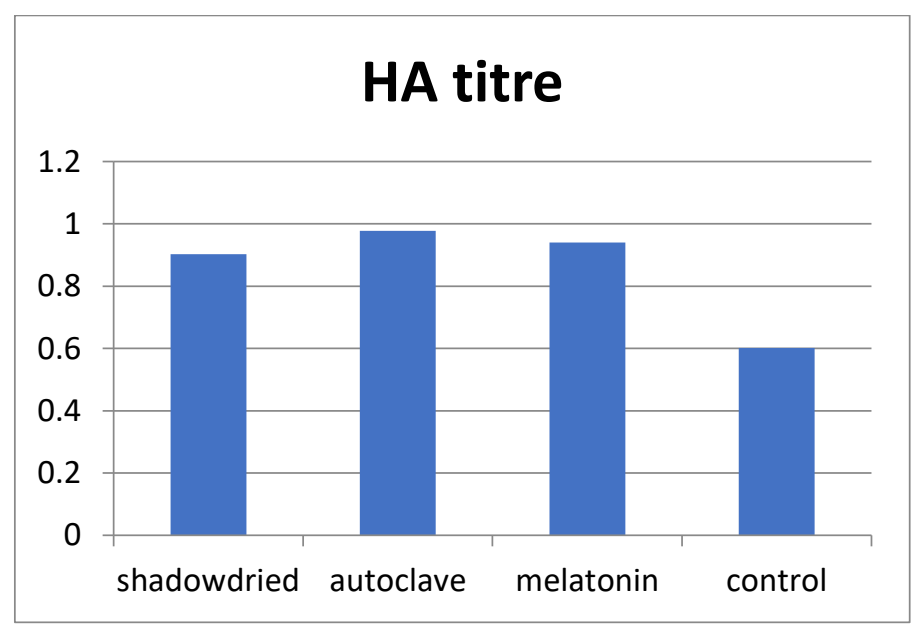

Figure 3: graph showing Hemagglutination titer value

Egg Quality

In the feeding experiment with Sargassum species of seaweed along with melatonin and a control group, the color of the egg yolk from the control group appeared somewhat paler than those of other groups. As figure 4 indicates a definite darkening of the color was observed for 
the egg yolk of the melatonin group. In the seaweed supplemented group, the shadow-dried group had better yolk coloration compared to autoclaved-dried group. This shows that other than melatonin injection (synthetic treated group), the sun-dried extract of seaweed showed deeper coloration than the control group. Thus, it can be concluded that seaweed contain many kinds of carotenoid pigments (Shinichi Takaichi, 2011) ${ }^{17}$ and when aqueous extract of Sargassum species of seaweed supplementation is fed as a poultry feed additive, the pigments are transferred to their by-product, making the color of egg yolk darker and more pigmented. Thus, enriching the nutritional quality of the egg.

(Jensen, A., 1962) ${ }^{18}$, conducted a study on the effect of seaweed carotenoids on egg yolk coloration and proved that, upon addition of $10-15 \%$ of seaweed meal to the basil diet of poultry can increase the carotenoid content of the yolk and Results of the present study pertaining to effect of seaweed in yolk coloration are in agreement to the findings of Ross and Jensen. In another review (Hftie and Sannam, 1960) ${ }^{19}$ found a significant color different in the yolk when the livestock were fed with $5 \%$ of seaweed supplementation. However, this study showed that incorporation of Sargassum spp. Of seaweed as a feed supplementation is considered effective way in enhancing the yolk color of Vanaraja birds.

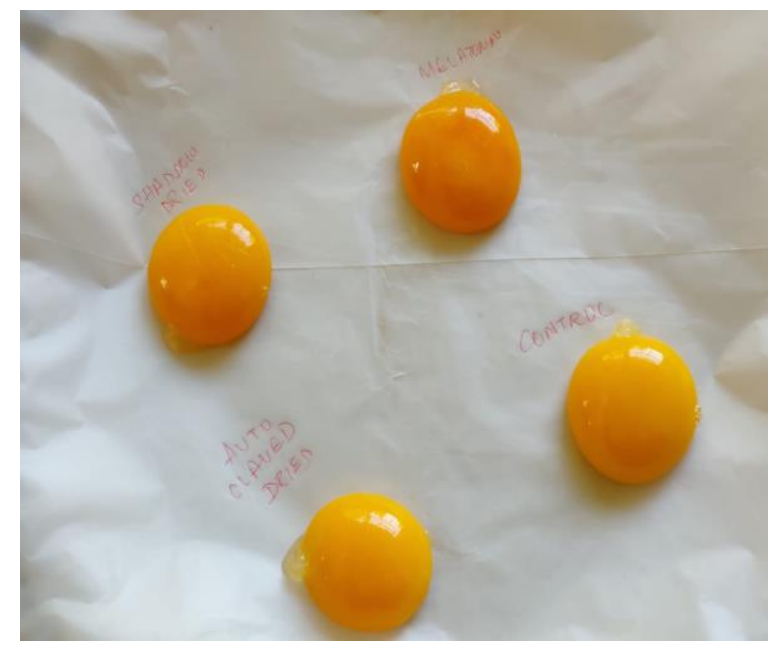

Figure 4: Effect of seaweed supplementation on egg yolk.

\section{CONCLUSION}

This study highlights the research on seaweed as a valuable and sustainable feed additive for poultry farming system. The study also revealed that the seaweed supplementation can contribute to enhance the antioxidant content in the chicken, in terms of immunity the aqueous autoclave extract has proven effective in increasing antibody titer value against GRBC antigens. Feeding Brown seaweed Sargassum species to the poultry improved the egg yolk color, the yolk from shadow-dried group has color similar to melatonin (synthetic drug) but was darker than the autoclaved-dried and the control supplemented group. This study concludes that the nutritional value of seaweed and their effects in animal feed/supplement can be used as an alternative to melatonin and other antibody growth promoter, thus utilization of seaweed in animal feed will help improve the animal food safety and security and could fulfill the 
requirement of meeting the essential adequate nutrients for the human health and well-being. This study also demonstrates the previous finding of novel use of seaweed and their effect on the livestock, therefore further study is expected to focus more on exploring different varieties and species of seaweed from across the world and their application at commercial level.

\section{REFERENCE}

1. Anne Mottet and G. Tempio. Global Poultry production: current state and future outlook and challenges. World's science journal. April 1, 2017.

2. Suleyman CALISLAR, Beyham YETER, Ahmed SAHIM. Importance of melatonin on poultry, Tarin doga dergisi, 2018.

3. Yaxiong Jia, Minghini Yang, Kuanfeng Zhu, Liang Wang, Yukun Song, Jing Wang, Wenxiang Qin, Zhiyuan $\mathrm{Xu}$, Yuchen and Guoshiliu. Melatonin in plantation of the egg-laying rate and quality in hens past their peak egg-laying age. Scientific report, 2016.

4. Times of India. What is seaweed, benefits and how to use it, June 22, 2020.

5. Leslie Mendoza Temple, ABOIM, Pooja Saigal. Hypothyroidism. Integrative Medicine (Fourth edition), 2018

6. Hemaiswarya Shanmugam, Ramaraj Sathaisivana, Raja Rathinam, Kulanthaiyesu Arunkumar, Isabel S. Carvalho. Algal biotechnology: An update from industrial and medical point of view, Omics Technologies \& Bioengineering. 2018.

7. Tiago Morais, Ana Inacio, Tiago Continho, Mariana Ministro, Joao Cotas, Leonel Pereira, and Kiril Bachcevandziev. Seaweed Potential in animal feed: A review. Journal of Marine Science and Engineering 25 July 2020.

8. Garima Kulshreshtha, Alan Critchley, Bruce Rathgerber, Glenn Stratton, Arjin H. Banskota, Jeff Hafting and Balakrishnan Prithiviaay. Animicrobial effect of selected cultivated red seaweed and their components in combination with tetracycline against poultry pathogen Salmonella Enteribacter. Journal of Marine Science and Engineering. 12July 2020.

9. Papadopoulou, A., Petrotos, K., Stagos, D., et al. Enhancement of antioxidant mechanism and reduction of oxidative stress in chickens after the administration of drinking water enriched with polyphenolic powder from olive mill waste water. Oxidative medicine and cellular longevity. 2017.

10. Stefaniak, T., Madej, J.P., Graczyk, S., Siwek, M., et al. Selected prebiotics and symbiotic administered in ovo can modify innate immunity in chicken broilers. BMC veterinary Research. 15, 105. 2019.

11. Martin, A., Gross, W.B. and Siegal, P.B. IgG and IgM responses in high and low antibody selected lines of chickens. Journal of Heredity, 80 (3): 249-252. 1989.

12. Fereshteh Zeraatpisheh, Farid Firouzbakhsh, Khosro Jani Khalili. Effect of macroalgae Sargassum angustifolium hot water exatracton hematological parameters and and immune responses in rainbow trout (Oncohrynchus mykiss) infected with Yersinia rukeri. Journal of applied Phycolgy. 2018.

13. Begum, R., Howlader, S., Rashid, A.N.M.N., Rafiquzzaman, S.M., et al. Antioxidant and signal- Modulating effect of brown seaweed- derived compounds against oxidative stressassociated pathology. Oxidative Medicine and cellular longevity, Vol. 2021. 
14. Arun Kumar De, A. Kundu, V. Vsantha Ruban, MS Kundu, S.Jayakumar and Jai Sundar. Comparative study, haematology of vanraja, nicobari fowl and their various F1 crosses under hot humid climate of A\&N Island. Journal of Applied Animal Research. 03April,2012.

15. Qingchao Shi, Xiaobo Wen, Dashi Zhu, Jude Juventus Aweya, Shebgkang Li. Protective effect of Sargassum hornei against ammonia stress in juvenile black sea bream, Acanthopagrus schlegelii. Journal of applied Phycology. 2019.

16. Qadri SSN, Biswas1 A, Mandal AB et al., Production performance, immune response and carcass traits of broiler chickens fed diet incorporated with Kappaphycus alvarezii. Journal of Applied Phycology 31:753-760. 2019.

17. Shinichi Takaichi. Carotenoid in algae: distributions, biosynthesis and functions. Mar Drugs. National Library of Medicine. 2011.

18. Jensen, A. The effect of seaweed carotenoids on egg yolk coloration. Norwegian Institute of Seaweed Research, NTH, Trondhein. 1962.

19. Hftie, J., and F. Sannan. Experiments with seaweed meals as supplements to rations for chicks and laying hens (II). Scientific Reports from The Agricultural College of Norway, 39: (19): 1-76. 1960 